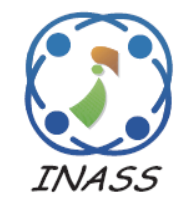

\title{
Multiple Linear Regression for Determining Critical Failure Factors of Agile Software Projects
}

\author{
Ahmed Abdelaziz ${ }^{1 *}$ \\ Nagy Ramadan Darwish ${ }^{2}$ \\ Hesham Ahmed Hefny ${ }^{3}$ \\ ${ }^{1,2}$ Department of Information Systems and Technology, Institute of Statistical Studies and Research, \\ Cairo University, Egypt \\ ${ }^{1}$ Department of Information Systems, Higher Technological Institute, Cairo, Egypt \\ ${ }^{3}$ Department of Computer and Information Sciences, Institute of Statistical Studies and Research, \\ Cairo University, Egypt \\ * Corresponding author's Email: Ahmed.aziz.1157@gmail.com
}

\begin{abstract}
The management of agile software projects plays an important role in the success or failure of projects. There are many factors of success or failure that clearly affect agile software projects. There is a big problem in not having a formal study to identify critical failure factors that assess the success or failure of projects accurately. This paper focuses on failure factors of agile software projects. This paper seeks to configure a formal study by using multiple regression analysis for identifying critical failure factors to avoid projects failure, thus saving time and money for the stakeholders. The proposed model is implemented by using $\mathrm{R}$ programming. The proposed model also is implemented by two models. The first model based on critical failure factors (CFF) of agile software projects. The second model based the failure factors of the proposed in the initial list (FFIL). The model of CFF outperforms on the model of FFIL in order to accuracy by $10.7 \%$ and random error ratio by $4.8 \%$. The results revealed that only 10 out of 19 hypotheses were supported, identifying seven critical failure factors for Agile software development projects: (a) Organizational culture too traditional, (b) Lack of agile logistical arrangements,(c) Lack of project management competence, (d) Lack of team work, (e) Bad customer relationship, (f) defined project scope, and (g) defined project planning.
\end{abstract}

Keywords: Agile software projects, Critical failure factors, Multiple linear regressions, R programming.

\section{Introduction}

Software projects have been important of many researches, organizations, and software developers in the last years. Software development has sometimes failed, thus often performing in delayed, failed and rejected software projects [1]. Even those software projects executed may require costly on-going maintenance and service packs.

The above shortages have influenced the bottom line of software development communities in a significantly $[2,3]$. The big challenge here is how software development management can be enhanced to avert the above problems of inaccurate and inefficiency? There has been a novel appearance of software development process called agile methods, which work rather differently from conventional methods.

Agile software development has many characteristics differently from traditional methods such as high quality, visibility, Small incremental releases, accommodating change due to volatile requirements, iterative releases, continuous integration, transparency and Predictable Costs [4]. Agile software development is based on several methods of which Scrum, Dynamic System Development Method (DSDM), Extreme Programming (XP), Lean Software Development (LD), Feature-Driven Development (FDD), Adaptive Software Development (ASD) and Crystal.

IT communities try to improve agile software projects by overcoming the reasons that lead to the 
Table 1. CHAOS report for agile software projects

\begin{tabular}{|l|c|c|c|c|}
\hline Size & Method & Successful & Challenged & Failed \\
\hline All Size Projects & Agile & $39 \%$ & $52 \%$ & $9 \%$ \\
\hline Large Size Projects & Agile & $18 \%$ & $59 \%$ & $23 \%$ \\
\hline Medium Size Projects & Agile & $27 \%$ & $62 \%$ & $11 \%$ \\
\hline Small Size Projects & Agile & $58 \%$ & $38 \%$ & $4 \%$ \\
\hline
\end{tabular}

failure of those projects. Many reasons have been recorded which led to failure of agile software projects such as poor management, lack of clarity of project plan, inefficiency of work team members and etc. Agile software projects have been evaluated by many success or failure factors. However, many agile software projects are still failing, according to the CHAOS report on the failure of many those projects, as shown below in Table 1 [5].

The critical failure factor approach to determine and gauge a project's performance in each release was first developed [6]. There has not been any official study on critical failure factors in the agile software development project per se, based on recent searches in peer revised academic work related to this topic. There is a big problem in not having a formal study to identify critical failure factors that assess the success or failure of projects accurately.

Many researchers have sought to find critical failure factors that affect agile software projects, but using traditional statistical methods. This paper uses intelligent techniques to determine and provide insight into the critical failure factors that help agile software projects to succeed. Intelligent techniques such as logistic regression and linear regression and others are used to configure a formal study that helps to identify critical failure factors in agile software projects.

This paper seeks to configure a formal study by using multiple regression analysis for identifying critical failure factors to avoid projects failure, thus saving time and money for the stakeholders. Therefore, the proposed model relies on regression analysis for selecting critical failure factors of agile software projects. The proposed model is applied on $\mathrm{R}$ studio. This paper also introduces overview of failure factors of agile software projects, and multiple regression analysis, as follows:

\subsection{Failure factors of agile software projects}

Failure factors of agile software projects are very important for stakeholders to identify and measuring an organization's performance. This paper seeks to collect the preliminary failure factors that influencing on agile software projects. It can be classified into four categories: organizational, people, process, and technical, as shown below in Table 2 .
Table 2. Preliminary failure factors that influence on agile software projects

\begin{tabular}{|c|c|}
\hline Dimension & Failure Factor \\
\hline $\begin{array}{c}\text { Organizational } \\
{[1]}\end{array}$ & $\begin{array}{ll}\text { 1. } & \text { Lack of executive } \\
\text { sponsorship(f1) }[11,18,20] \\
\text { 2. } \\
\text { Lack of management } \\
\text { commitment(f2) }[7,18,20] \\
\text { 3. } \begin{array}{l}\text { Organizational culture too } \\
\text { traditional(f3) }[12,18,20]\end{array} \\
\text { 4. } \begin{array}{l}\text { Organizational culture too } \\
\text { political(f4) }[9,18,20]\end{array} \\
\text { 5. } \begin{array}{l}\text { Organizational size too } \\
\text { large(f5) }[8,18,20]\end{array} \\
\text { 6. } \begin{array}{l}\text { Lack of agile logistical } \\
\text { arrangements(f6) }[10,18,20]\end{array}\end{array}$ \\
\hline $\begin{array}{r}\text { People } \\
{[2]}\end{array}$ & 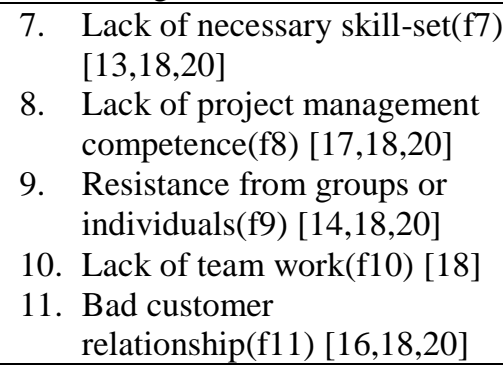 \\
\hline $\begin{array}{r}\text { Process } \\
{[3]}\end{array}$ & $\begin{array}{l}\text { 12. defined project scope(f12) } \\
{[15,18,20]} \\
\text { 13.defined project requirements(f13) } \\
{[19,18,20]} \\
\text { 14. defined project planning(f14) } \\
\text { [21,18] } \\
\text { 15. Lack of agile progress } \\
\text { tracking mechanism(f15) [22,27] } \\
\text { 16. Lack of customer } \\
\text { presence(f16) }[23,18,20] \\
17 \text { defined customer role(f17) } \\
{[24,30]}\end{array}$ \\
\hline $\begin{array}{r}\text { Technical } \\
{[4]}\end{array}$ & $\begin{array}{l}\text { 18. Lack of complete set of correct } \\
\text { agile practices(f18) }[25,26] \\
\text { 19. Inappropriateness of } \\
\text { technology and tools(f19) } \\
{[28,29]}\end{array}$ \\
\hline
\end{tabular}

\subsection{Multiple regression analysis}

Multiple regression analysis is composed of one dependent variable and many independent variables, but the dependent variable is persistent such as weight, height, number of hours, and etc $[8,9]$. In linear regression, the dependent variable (degree of effect in failure factors of agile software projects) has 
unlimited number of possible values. The coefficient interpretations of independent variables are straightforward. It uses ordinary least squares approach to reduce the errors and reach the optimal possible fit. It uses the general linear equation, as follows [10]:

$$
\mathrm{Y}=\mathrm{B} 0+\sum(\mathrm{BiXi})+\epsilon
$$

Where $\mathrm{Y}$ is a persistent dependent variable and independent variables $\mathrm{Xi}$ are usually persistent. $\mathrm{B} 0$ is the intercept (the value of $y$ when $x=0$ ). The slope of the line is Bi. $\epsilon$ is a term of the distinction that is not interpreted by the model and is usually just called "error".

This paper used the web survey process to collect data. A web survey with Likert scale questionnaires and demographic information combination was distributed to the target population. There were two sections in the survey. The first section was on demographic data, which included both the respondent's demographic information as well as the agile project information. The second section was on failure factors. To measure importance of failure factors, a 3-point Likert scale was used to reflect the level of perception of the question by the respondent. A survey was conducted among agile professionals, collecting survey data from 163 persons from 12 countries across the world.

This paper is arranged as follows: Section 2 introduces a related work. Section 3 introduces the proposed model. Section 4 introduces a proposed algorithm of the multiple regression analysis. Section 5 introduces experimental results and finally, section 6 presents conclusion and future work.

\section{Related work}

Previous work shows the importance studies that using statistical and intelligent techniques for analyzing success and failure factors of agile software projects, as follows:

$\mathrm{V}$. Lalsing and et al, introduced a method to determine critical factors in people dimension of software projects by using statistical techniques. This paper aims to evaluate people factors to select the suitable number of team in each project. This process can affect the performance of the projects in order to time and cost [11]. This paper is based on traditional methods such as time series. This paper depends on the people dimension despite the existence of four dimensions interested in agile software projects which are people, organization, technique and process. Researcher opinion, this paper can determine the critical factors in people dimension of software projects.

H. TAHERDOOST and et al, introduced an approach to evaluate IT project success/failure factors and determining the associated risks. This paper seeks to find the optimal success/failure factors of IT projects to prevent failure of it [12]. This paper is based on mean square error for determination risk factors in IT projects. This method is not enough to determine critical risk factors in IT projects. Researcher opinion, lack of clarity the failure factors that effect on IT projects.

S. Lee and et al, introduced a framework to evaluate agile software development in small projects based on success factors. This paper aims to obtain a suitable success factors to help small agile software projects to succeed [13]. This paper is based on success factors that have impact small software projects by statistical methods. This paper is based on two dimensions from four dimensions which are people and technique. Researcher opinion, this paper can evaluate agile software projects based on suitable success factors.

M. Tanner and et al introduced a new method to determine critical success/failure factors of agile software projects and compared it to traditional methods such as waterfall model. This paper shows that the agile method outperforms on waterfall model in software projects [14]. This paper is based on two dimensions to determine critical success/failure factors by statistical methods. Researcher opinion, agile method can affect the software projects more than traditional method such as waterfall model.

K. M. B. Silva and et al, introduced a survey study to determine a critical success/failure factors of agile software projects. Critical factors can be classified into three dimensions: people, process and technology [15]. This paper is based on three dimensions to determine critical success/failure factors by statistical methods. This paper is based on questionnaires by experts, leading to inaccurate results. Researcher opinion, this paper can determine critical success/failure factors of agile software projects.

R. Amponsah and et al, introduced a study to find a positive relationship between success factors and agile software projects. This paper aims to determine critical success factors that influencing on agile software projects [16]. This paper is based on poor parameters. There is over fitting, missing and incomplete data. Researcher opinion, this paper can find critical success factors of agile software projects.

S. Maierhofer and et al, introduced a study to evaluate the effect of agile method on success of software projects. This paper aims to evaluate the 
relation between change in requirements and agile methods on project succeed. This paper also uses regression models to measure project succeed in order to requirement changes and efficient execution [17]. This paper cannot find evidence that two dimensions of agile software projects are highly correlated. Researcher opinion, this paper has succeeded to find the optimal relation between change in requirements and agile methods on project succeed.

T. Chow and et al, introduced a survey study to evaluate critical success factors of agile software project by using regression models. Critical success factors have been evaluated by four categories: Quality, Scope, Time, and Cost. The results show that only 10 out of 48 hypotheses were supported, identifying three critical success factors for agile software development projects: (a) Delivery Strategy, (b) Agile Software Engineering Techniques, and (c) Team Capability [18]. This paper is based on regression model, but there is incomplete and missing data. This problem makes the results lose their accuracy. Researcher opinion, this paper has succeeded to determine critical success factors of agile software projects and providing three significant critical success factors of them.

N. Cerpa and et al, introduced a new model to predict success of agile software project by using logistic regression analysis. This paper aims to enhance accuracy of the prediction success of agile software projects. The proposed model outperforms on traditional statistics models [19]. Logistic regression has many defects such as identifying independent variables, limited outcome variables, over fitting the model and etc. Researcher opinion, the proposed model has succeeded to predict success of agile software projects by using logistic regression analysis.

D. Stankovica and et al, introduced a study to determine critical success factors of agile software project in IT companies by using regression models. Critical success factors have been evaluated by four categories: Quality, Scope, Time, and Cost. The results show that only 5 out of 48 hypotheses were supported, identifying three critical success factors for agile software development projects: (a) Project definition process, (b) Project nature, and (c) Project schedule [20]. Regression model is assumed that the cause and effect relationship between the variables remains unchanged. This problem in the regression model makes the results unreliable. Researcher opinion, this paper has succeeded to determine critical success factors of agile software projects in IT companies and providing three significant critical success factors of them.
V. Montequin and et al, introduced the framework to find critical success factors of agile software projects in information and communication technology in Spain. This paper aims to determine critical success/failure factors that affect agile software projects in information and communication technology domain. The results show that the five of significant success factors are clear vision and goals, realistic cost and time estimation, reachable goals and expectations, fluent communication among all stakeholders, and clear specification of project's requirements. The results also show that the five of significant failure factors are customer requirement inaccurate, continuous changes to initial requirements, inaccurate time estimations, inaccurate cost estimations, and badly defined specifications [21]. This paper is based on statistical method such as estimating a mean. This method has many conditions such as data approximately normal, large sample size and etc. This framework did not meet all the required conditions in the proposed statistical method. Researcher opinion, the proposed framework can find the critical success/failure factors of agile software projects in information and communication technology.

D. S. Nguyen and et al, introduced the study to determine critical success factors of agile software project in IT companies. This paper aims to find positive success factors that may be able to improve project management strategies and cost benefits leading to higher efficiency, profitability, and productivity thus benefiting management, employees, and customers [22]. This paper is based on statistical method such as Test to Compare Two Means. This method has many conditions such as Independent samples from the two populations, large sample size and etc. This approach did not meet all the required conditions in the proposed statistical method. Researcher opinion, positive success factors help to enhance IT project management strategies.

S. L. Lam and et al, introduced the approach to determine critical success factors of agile software project in IT projects. Critical success factors have been evaluated by regression models. The factors that significantly influence project success are: project management process, project definition process, customer involvement, and management commitment [23]. Researcher opinion, critical success factors can effect on IT project success.

K. Jammalamadaka and et al, introduced a study to select appropriate agile models such as Extreme programming, Scrum and others for applying of them on software projects. This paper aims to enhance performance of software projects through agile models in order to achieve customer satisfaction [24]. 
This paper is based on statistical method such as Relationship in a 2-Way Table. This method has many conditions such as all expected counts should be greater than 1, At least $80 \%$ of the cells should have an expected count greater than 5 and etc. This approach did not meet all the required conditions in the proposed statistical method. Researcher opinion, this study can find suitable agile models for using of them on software projects.

K. N. Rao and et al, introduced the study to measure the effect of agile models on software projects. This paper aims to evaluate agile models in order to success/failure factors for applying of them on software projects. Selecting the optimal agile model works to help the software project to succeed [25]. This paper is based on statistical method such as Test about a Slope. This method has many conditions such as the form of the equation that links the two variables must be correct, the errors terms have equal variances, the error terms are independent of each other and etc. This approach did not meet all the required conditions in the proposed statistical method. Researcher opinion, this study can find the optimal agile models for assisting the software projects to succeed.

P. Serrador and et al, introduced an approach to determine success factors that affect the project success by using quantitative analysis. This paper seeks to configure a formal study to find positive success factors for assisting projects to succeed [26]. Quantitative approach cannot account for nonnumerical information. Researcher opinion, the proposed approach can determine success factors that affect the success of projects.

W. Jawaid and et al, introduced a new model to extract success factors from the agile development practices that are successful in the software industry. The proposed model relies on data mining techniques especially fuzzy- c mean, multiple linear regressions, clustering and etc. the results show that the proposed model (fuzzy- c mean) outperforms on data mining techniques for determining success factors of agile software projects [27]. This paper is based on data mining techniques to predict success of agile software projects. This paper describes the factors of success and failure that affect the agile software projects. Researcher opinion, the proposed model is the optimal technique to extract success factors that affect the success of software projects.

R. Ibrahim and et al, introduced a method to determine failure factors that affect the failure of agile software project in IT companies. This paper aims to find the positive failure factors to avoid the failure of IT projects. This study classifies the failure factors into four critical factors: Poor top management support, Poor consultant effectiveness, poor project management effectiveness and Lack of User Involvement [28]. This paper is based on statistical method such as Test to Compare Several Means. This method has many conditions such as independent samples from the $\mathrm{t}$ populations, equal population standard deviations and etc. This approach did not meet all the required conditions in the proposed statistical method. Researcher opinion, this paper has succeeded to determine the critical failure factors to avoid the failure of IT projects.

V. N. Vithana and et al, introduced a framework to determine critical success factors that influence on the software project success by using statistical data analysis. This paper aims to find critical success factors for assisting software projects to succeed. The results show that the critical success factors are customer satisfaction, customer commitment, team size, corporate culture, technical competency, decision time, customer commitment and training and learning have an influence on the success of the software projects [29]. This paper is based on statistical method such as Test of Strength \& Direction of Linear Relationship of 2 Quantitative Variables. This method has many conditions such as 2 variables are continuous, No significant outliers, linear relationship between the variables and etc. This framework did not meet all the required conditions in the proposed statistical method. Researcher opinion, the proposed framework has succeeded to find critical success factors that affect the success of projects.

S. Abdalhamid and et al, introduced the study to find success/failure factors of agile software projects. This paper aims to determine positive success/failure factors to avoid failure of the software projects. There are many success/failure factors that influence on the success of projects such as customer participation and bad relationship with the customer [30]. This paper is based on statistical method such as Test to Compare Two Population Variances. This method has many conditions such as independent samples from the 2 populations and etc. This approach did not meet all the required conditions in the proposed statistical method. Researcher opinion, this study has succeeded to find critical success/failure factors that influence on the success of projects.

Through related work, most researches have not formal study to find critical failure factors of agile software projects. Most researches is based on two or three dimensions from four dimensions in agile software projects, but the proposed method is based on four dimensions which are people, process, technique and organizational. Critical failure factors 


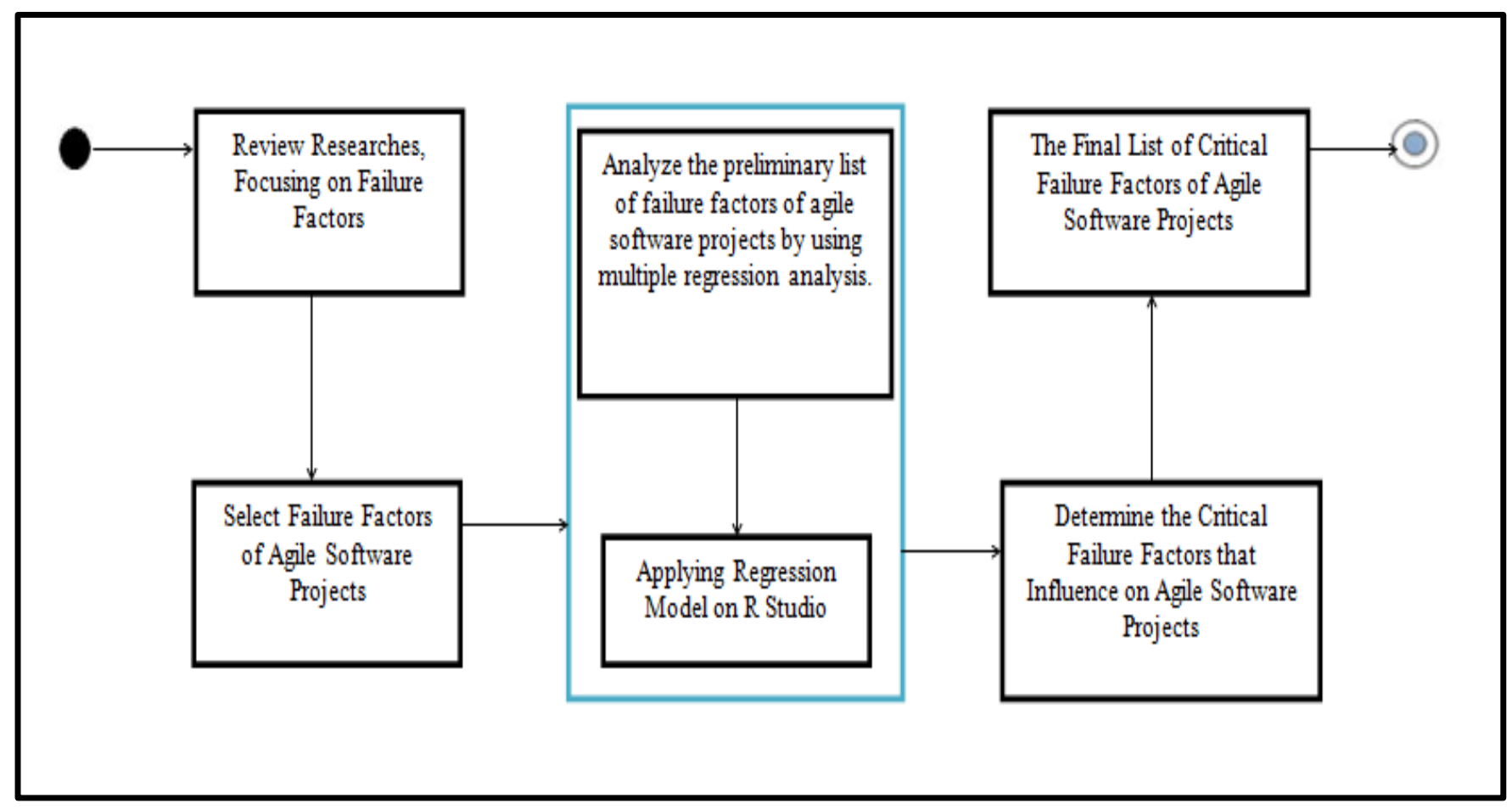

Figure. 1The proposed regression model to determine critical failure factors of agile software projects

seek to support agile software projects to avoid failure of them. This paper tries to configure a formal study to find critical failure factors for helping agile software projects to succeed.

This paper also proposes an intelligent model to determine critical failure factors of agile software projects efficiently.

\section{The proposed model}

This section introduces the proposed model for determining critical failure factors of agile software projects. The proposed model is composed of three parts: as shown below in Fig. 1.

1. Review the related work to find important failure factors regarding to agile software projects.

2. Analyze the preliminary list of failure factors of agile software projects by using multiple regression analysis.

3. Determine the final list of critical failure factors of agile software projects.

Fig. 1 introduces the activity diagram to determine critical failure factors of agile software projects. The proposed model is composed of three main parts. The first part aims to determine the important failure factors of agile software projects and selecting the critical failure factors that influence on agile software projects. Critical failure factors were selected based on some evaluation criteria such as ambiguity, usability and agility. The proposed model analyzes the preliminary list of failure factors of agile software projects by using multiple regression analysis to determine critical failure factors of agile software projects. Finally, the proposed model can determine the final list of critical failure factors that are helping the organizations to avoid failure of agile software projects to save time and cost. The proposed model was applied on $\mathrm{R}$ studio.

\section{The proposed algorithm of the multiple regression analysis}

This section introduces an algorithm for determining the critical failure factors that influence on agile software projects by multiple regression analysis. Fig. 2 shows the flow chart of the proposed algorithm. Multiple regression analysis introduced regression statistics such as standard error (SE), Rsquared (RS), adjusted R-squared (ARS) and P-value (PV). SE shows a first handle on how fully the provided equation fits the specimen data. It is critical to the units of gauge of the dependent variable. RS shows the explanatory force of the regression model. ARS is an updated version of RS that has been modified for the set of predictors in the model. It increases only if the novel terms enhance the model more than would be anticipated. It decreases when a predictor enhances the model by less than anticipated. 
It is always lower than the RS. PV helps to determine the importance of the statistical results. It is a number between 0 and 1 and explicated in the following method: a small PV (typically $\leq 0.05$ ) indicates strong evidence against the null hypothesis. The null hypothesis is rejected from the statistical results. The steps of the proposed algorithm are as follows:

Algorithm 1. The proposed algorithm of the multiple regression analysis to determine critical

failure factors that influence on agile software projects

1. Input : $\alpha<$ (dependent variable degree of effect the failure factors of agile software projects)

2. $\sigma<$ (independent variables failure factor that influence on agile software projects)

3. Output : $\beta \leftarrow$ (critical failure factors list of agile software projects)

4. MLR $=$ Multiple Linear Regression

5. SSR $=$ Sum of Squares Regression

6. SST $=$ Sum of Squares Total

7. $n=$ The Number of Data Points

8. $k=$ The Number of Parameters

9. $F i=$ the prediction value

10. $Y i=$ the true value

11. $\dot{Y}=$ the mean of $Y i$

12. $\mathbf{S S R}=\sum_{\mathrm{i}}(\mathbf{F i}-\dot{Y})^{2}$

13. $\mathrm{SST}=\sum_{\mathbf{i}}(\mathbf{Y i}-Y)^{2}$

14. $\mathrm{SE}=$ Standard Error

15. RS = R- Squared

16. ARS $=$ Adjusted R- Squared

17. $\mathbf{P V}=\mathbf{P}$-value

18. Build the MLR model based on the set of $\alpha$ and $\sigma$

19. Estimate the MLR model

20. Check the value of SE. SE is calculated as follows:

$$
\mathrm{SE}=\sqrt{\frac{\operatorname{SSR}}{n-2}}
$$

21. Check the value of RS. RS is calculated as follows:

$$
\mathrm{RS}=1-\frac{S S R}{S S T}
$$

22. Check the value of ARS. ARS is calculated as follows:

$$
\operatorname{ARS}=1-\frac{S S R /(n-k-1)}{S S T /(n-1)}
$$

23. If $(A R S<0.5)$

24. Change the explanatory of failure factors

25. Go to step 18

26. Else

27. Approve the model

28. End If

29. Check PV for each variable to determine $\beta$

30. If $(\mathrm{FW}<0.05)$

31. Approve $\beta$

32. Else

33. Refuse the other failure factors

34. End If

35. Return $\beta$

\section{Experimental results}

In our experiment, it is an exploratory experiment to detect which features can positively affect the failure of agile software projects. This experiment is used a multiple regression analysis, where the relationship between multiple independent variables (failure factors in agile software projects) and the dependent variable (degree of effect the failure factors in agile software projects) is specified. Based on the Eq. (2), the multiple regression analysis can be determined as follows:

$$
\mathrm{Y}=\beta 0+\beta 1 \mathrm{x} 1+\beta 2 \times 2+\ldots .+\beta 7 \mathrm{x} 7+\varepsilon
$$

- $Y$ : is degree of effect the failure factors in agile software projects

- $\beta 0$ : is the y-intercept

- $\beta \mathrm{i}$ : is the regression coefficient

- Xi: critical failure factors

- $\varepsilon$ : the random error term

The experiment of the proposed model is implemented by a set of consecutive steps. First, the dataset attributes are divided into dependent and independent variables (refer to Table 2). Then, the dataset is composed of $70 \%$ training data and $30 \%$ testing data. The proposed model is used the ordinary least squares that suppose sturdy linear relationship between the independent variables and the dependent variable. After that, L2 Regularization is used to improve the quality of the proposed model. 


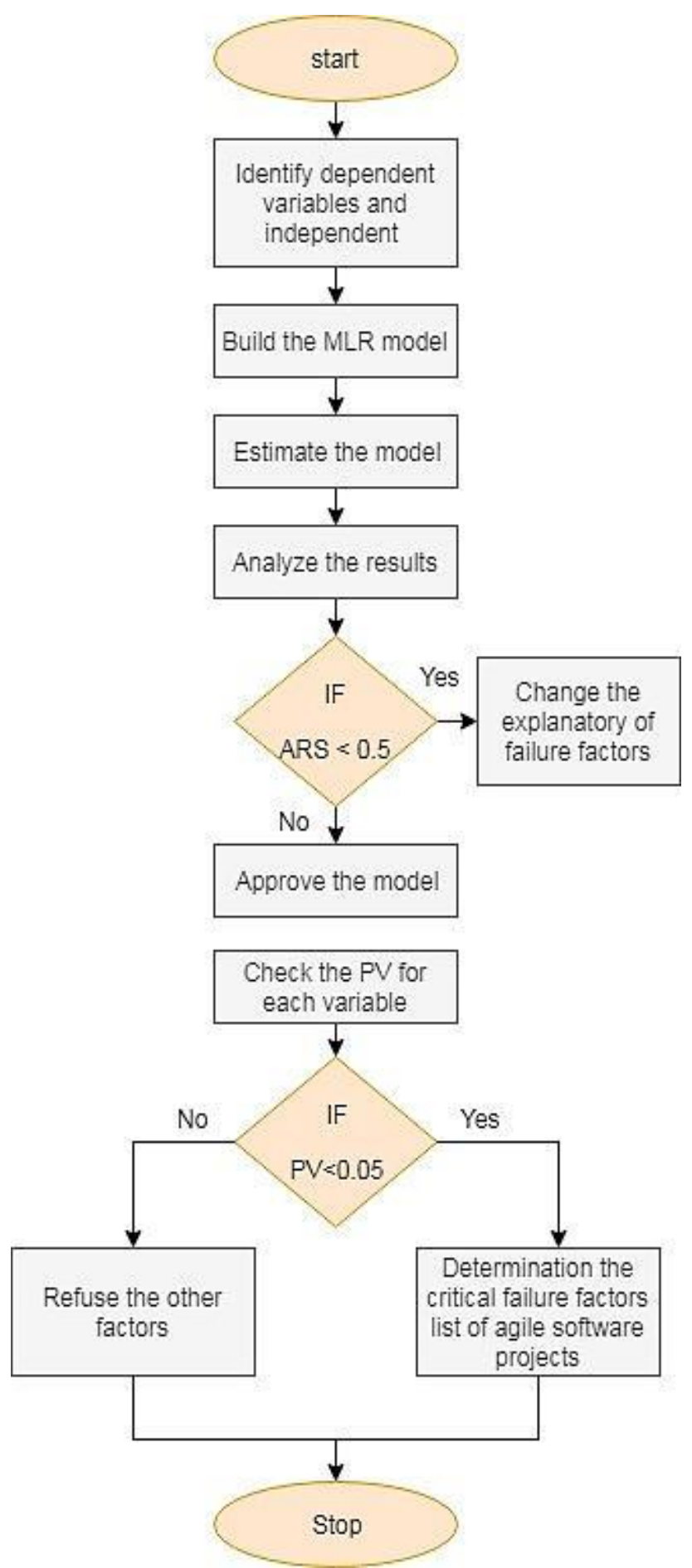

Figure. 2 Flow chart of the proposed algorithm for MLR model

Table 3. Summary of regression statistics

\begin{tabular}{|c|c|}
\hline \multicolumn{2}{|c|}{ Regression Statistics } \\
\hline RS & 0.803 \\
\hline SE & 0.1518 \\
\hline ARS & 0.7981 \\
\hline
\end{tabular}

In our experiment of the proposed model, there are two important tables introduced to show a significant data about the proposed model and the contribution of each independent variable to the regression model. Table 3 shows the regression statistics of the proposed model, as shown in Fig. 3. This table provides important information such as RS which is equal to $0.803, \mathrm{SE}$ is equal to 0.1518 and finally, ARS is equal to 0.7981 . This articulates that $79.8 \%$ of the discrepancy in the dependent variable (degree of effect the failure factors in agile software projects) can be interpreted by the independent variables (failure factors in agile software projects), while the rest $(20.2 \%)$ is interpreted by other causes. This means that the proposed model is agreeable and statistical important to define the most significant features that affect the selection of failure factors of agile software projects. In other hand, the failure factors selection process of agile software projects is robustly related to the selected categories of people, organizational, process, and technical.

Fig. 3 shows the coefficients analysis that articulate the individual contribution of each independent feature to the proposed model. The PV in the Coefficients figure articulates the important

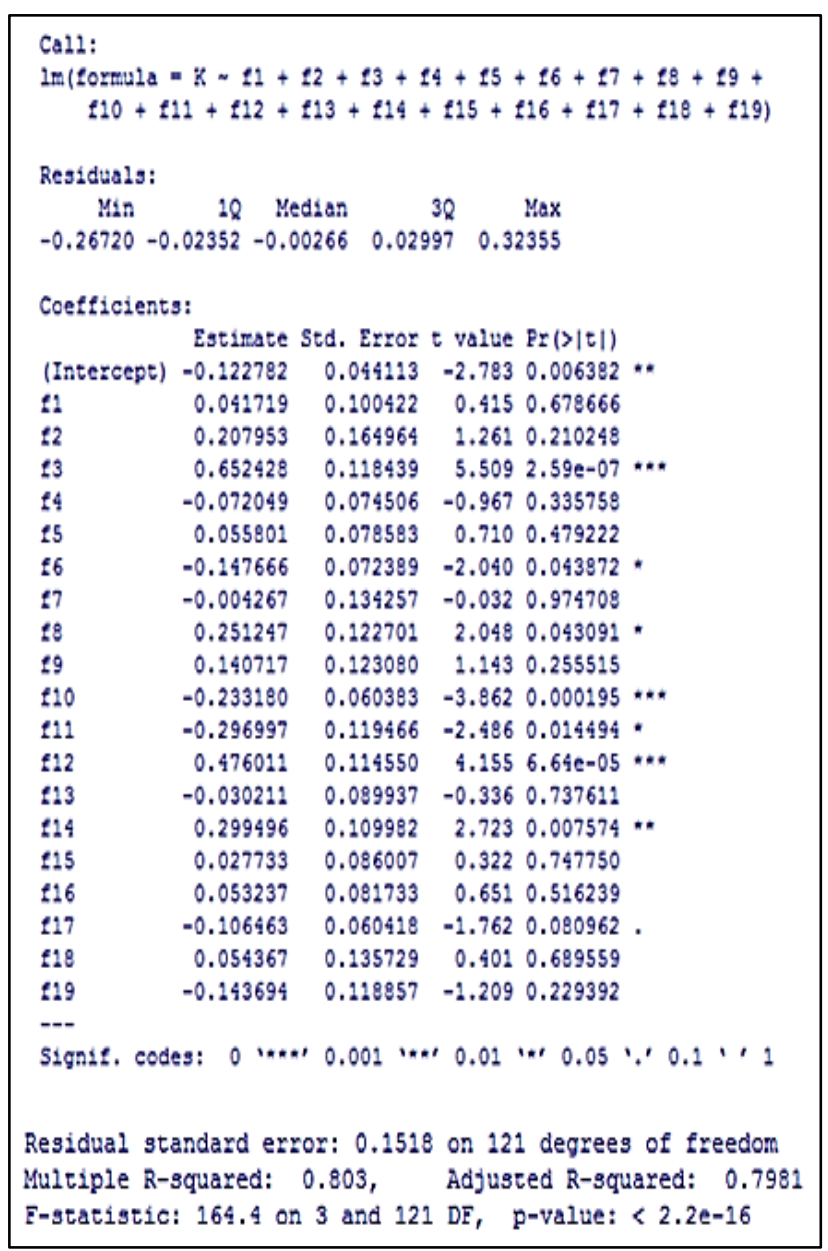

Figure. 3 Coefficients analysis based initial list of failure factors by using $\mathrm{R}$ programming 
Table. 4 Summary of critical failure factors of agile software projects

\begin{tabular}{|c|c|c|c|c|}
\hline No & Factor ID & Factor Name & PV & Dimension \\
\hline 1 & F3 & Organizational culture too traditional & 0.000259 & Organizational \\
\hline 2 & F6 & Lack of agile logistical arrangements & 0.043872 & Organizational \\
\hline 3 & F8 & Lack of project management competence & 0.043091 & People \\
\hline 4 & F10 & Lack of team work & 0.000195 & People \\
\hline 5 & F11 & Bad customer relationship & 0.014494 & People \\
\hline 6 & F12 & defined project scope & 0.00664 & Process \\
\hline 7 & F14 & defined project planning & 0.007574 & process \\
\hline
\end{tabular}

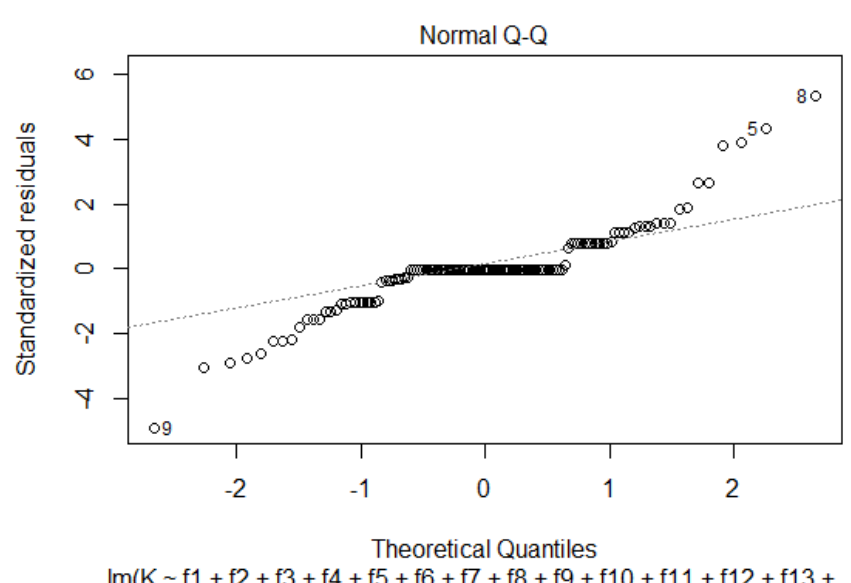

Figure. 4 Natural distribution of agile data set After the sifting of PV-values, the most critical features which influence the failure factors selection were specified as introduced in Table 4.

independent features that effect on failure of agile software projects. If $\mathrm{PV}<0.05$, the coefficient is statistically important. For instance, the PV for f11 $(p=0.01)$ is less than 0.05 , therefore, this feature should be accepted. For that, those features with importance level $(\mathrm{PV}<0.05)$ and top coefficient values would be recognized as elects for being significant features influencing failure factors selection.

Fig. 4 shows the points around the line. Thus, the data used is distributed according to natural distribution. Therefore, the data of independent factors is highly effect on the dependent factor.

The model was reconstructed using critical failure factors to improve the proposed model accuracy. The proposed new model was improved as shown in Fig. 5 where the model accuracy is $90.5 \%$. The random error rate was reduced to $10.4 \%$.

The model based on critical failure factors (CFF) outperform on the model based the failure factors of the proposed in the initial list (FFIL) in order to the accuracy and random error ratio, as shown below in Figs. 6 and 7.
Call:

$\operatorname{lm}($ formula $=\mathrm{K} \sim \mathrm{f} 3+\mathrm{f} 6+\mathrm{f} 8+\mathrm{f} 10+\mathrm{f} 11+\mathrm{f} 12+\mathrm{f14})$

Residuals:

Min 18 Median 30 Max

$\begin{array}{llllll}-0.29177 & 0.00046 & 0.00046 & 0.03883 & 0.41783\end{array}$

Coefficients:

Estimate Std. Error t value $\operatorname{Pr}(>|t|)$

(Intercept) $-0.16600 \quad 0.03650 \quad-4.548 \quad 1.33 \mathrm{e}-05 * * *$

f3 $\quad 0.60666 \quad 0.06807 \quad 8.912 \quad 7.86 \mathrm{e}-15 * * *$

$\begin{array}{llllll}\text { f6 } & -0.15259 & 0.05991 & -2.547 & 0.01217 \text { * }\end{array}$

$18 \quad 0.42612 \quad 0.06169 \quad 6.9082 .74 \mathrm{e}-10 k * *$

f10 $\quad-0.26606 \quad 0.05045 \quad-5.274 \quad 6.19 e-07 * * *$

$\begin{array}{lllll}f 11 & -0.24789 & 0.09852 & -2.516 & 0.01322 *\end{array}$

$112 \quad 0.57544 \quad 0.07169 \quad 8.026 \quad 8.73 \mathrm{e}-13 * * *$

f14 $\quad 0.30678 \quad 0.09695 \quad 3.164 \quad 0.00198$ k*

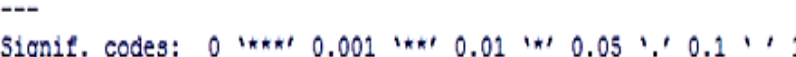

Residual standard error: 0.1039 on 105 degrees of freedom Mulciple R-squared: 0.9199 , Adjusted R-squared: 0.9054 F-statistic: 63.49 on 19 and 105 DF, p-value: $<2.2 \mathrm{e}-16$

Figure. 5 Coefficients analysis based critical failure factors by using R programming

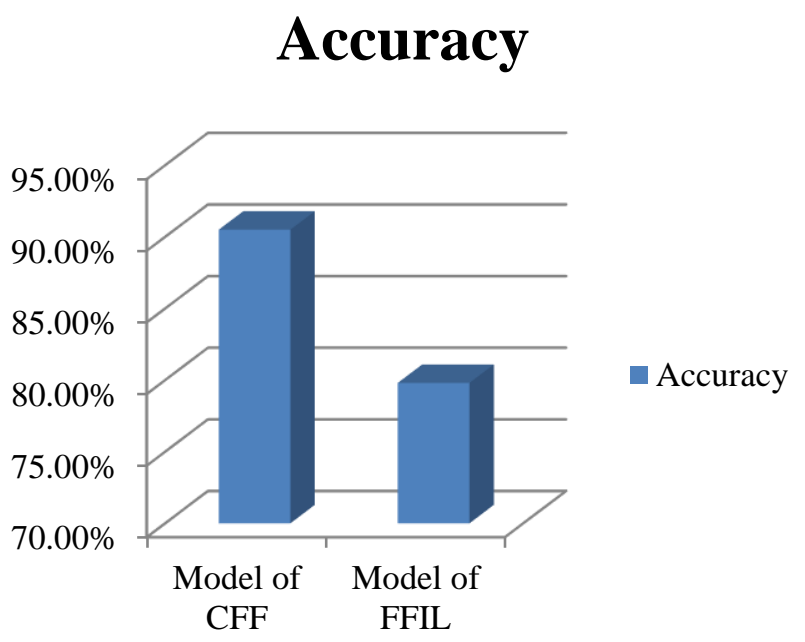

Figure. 6 The comparison between model of CFF and model of FFIL in order to accuracy 


\section{Random Error Ratio}

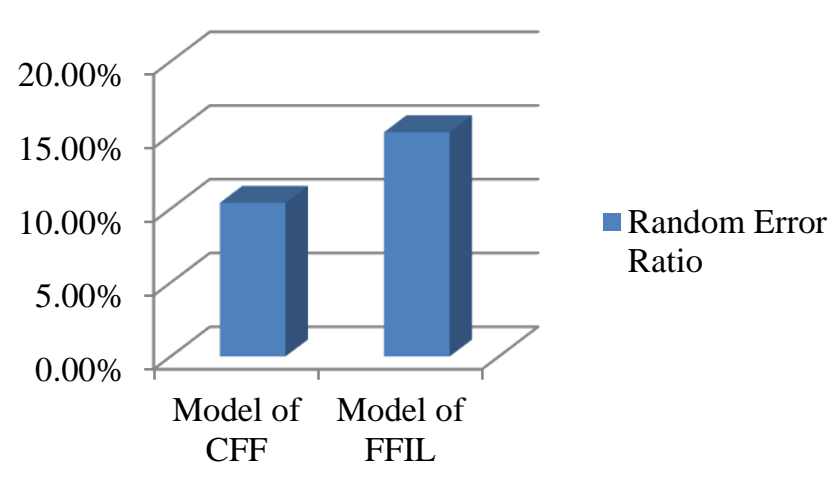

Figure. 7 The comparison between model of CFF and model of FFIL in order to random error ratio

\section{Accuracy}

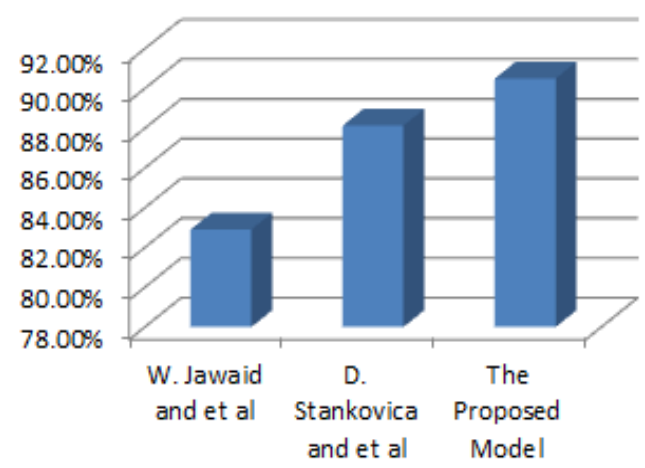

Figure. 8 The accuracy of the proposed model compared to the state of the art models

The model of CFF outperforms on the model of FFIL in order to accuracy by $10.7 \%$ and random error ratio by $4.8 \%$.

The accuracy of the proposed model outperforms on the state-of-the art methods as shown in Fig. 8.

The proposed model outperforms on the state of the art models in terms of the number of dimensions that are using to determine critical factors in agile software projects as shown in Fig. 9 and Table 5.

Table 5. The number of dimensions of the proposed model compared to the state of the art models

\begin{tabular}{|l|l|c|l|}
\hline No & Author's & $\begin{array}{c}\text { No of } \\
\text { Dimension }\end{array}$ & Dimensions \\
\hline 1 & V. Lalsing & 1 & {$[2]$} \\
\hline 2 & H. Taherdoost & 2 & {$[2,4]$} \\
\hline 3 & K. Silva & 3 & {$[2,3,4]$} \\
\hline 4 & R. Ibrahim & 1 & {$[4]$} \\
\hline 5 & $\begin{array}{l}\text { The Proposed } \\
\text { Model }\end{array}$ & 4 & {$[1,2,3,4]$} \\
\hline
\end{tabular}

\section{Number of Dimensions}

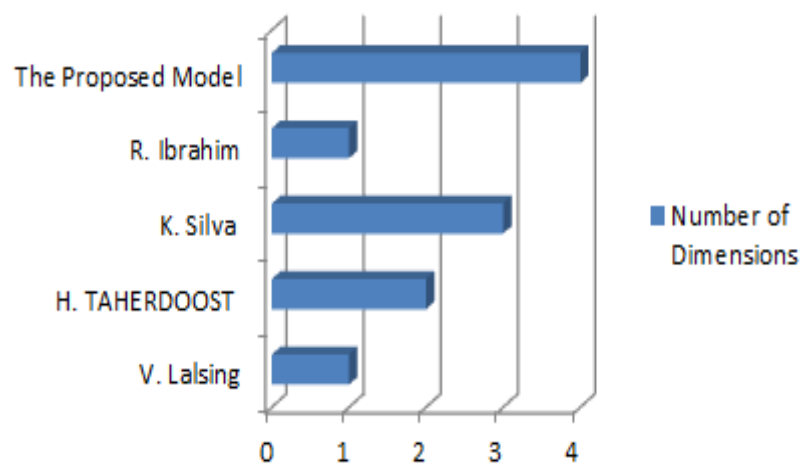

Figure. 9 The comparison between number of dimensions

\section{Conclusion and future work}

There is a major problem facing stakeholders in determining critical failure factors that affect agile software projects. This paper succeeded in determining critical failure factors of agile software projects by using multiple regression analysis. The proposed model is applied on $\mathrm{R}$ studio. The proposed model also is implemented by using two models, which are CFF and FFIL. The accuracy of CFF model is $79.8 \%$, and random error ratio is $15.2 \%$. The accuracy of FFIL model is $90.5 \%$, and random error ratio is $10.4 \%$. For that, the CFF model outperforms on FFIL model in order to accuracy and random ratio error. This paper succeeded in identifying 7 factors out of 19 factors that may affect the failure of agile software projects. Subsequently, reliability analysis and factor analysis were conducted to consolidate this preliminary list into a final set of 7 possible critical failure factors for each of the four project failure categories: organizational, people, process, and technical. It is recommended as a future work to use other intelligent techniques such as logistic regression in order to enhance the model accuracy and determining critical failure factors of agile software projects.

\section{References}

[1] I. Stamelos and P. Sfetsos, "Agile Software Development Quality Assurance", Information science references, Idea Group Inc, 2007.

[2] V. Jyothi and K. Rao, "Effective Implementation of Agile Practices", International Journal of Advanced Computer Science and Applications, Vol.2, No.3, pp. 41-48, 2011.

[3] E. Lzamly and B. Hussin, "An Enhancement of Framework Software Risk Management Methodology for Successful Software Development", Journal of Theoretical and 
Applied Information Technology, Vol. 62, No. 2, pp. 410-423, 2014.

[4] M. Shepperd and S. MacDonell, "Evaluating prediction systems in software project estimation", Information and Software Technology, Vol. 54, pp. 820-827, 2012.

[5] N. Darwish, A. Mohamed, and A. Abdelghany, "A Hybrid Machine Learning Model for Selecting Suitable Requirements Elicitation Techniques", International Journal of Computer Science and Information Security, Vol. 14, No. 6, pp. 1-12, 2016.

[6] F. Batarseh and A. Gonzalez, "Predicting failures in agile software development through data analytics", Software Quality Journal, Vol. 26, No. 1, pp. 49-66, 2018.

[7] R. Mohanty, G. Sahoo, and J. Dasgupta, "Identification of Risk Factors in Globally Outsourced Software Projects using Logistic Regression and ANN", International Journal of Sup. Chain. Mgt., Vol. 1, pp. 2-11, 2012.

[8] T. Pushpavathi, V. Suma, and V. Ramaswamy, "Defect Prediction in Software Projects-Using Genetic Algorithm based Fuzzy C-Means Clustering and Random Forest Classifier", International Journal of Scientific \& Engineering Research, Vol. 5, pp. 888-898, 2014.

[9] A. Mohamed, N. Darwish and H. Hefny, "Towards a Machine Learning Model for Predicting Failure of Agile Software Projects", International Journal of Computer Applications, Vol. 168, No. 6, pp. 20-26, 2017.

[10] T. Hovorushchenko and A. Krasiy, "Realization of the Neural Network Model of Prediction of the Software Project Characteristics for Evaluating the Success of its Implementation", International Conference on Intelligent Data Acquisition And Advanced Computing Systems, pp. 348-353, 2015.

[11] V. Lalsing, S. Kishnah, and S. Pudaruth, "People Factors in Agile Software Development and Project Management", International Journal of Software Engineering Applications, Vol. 3, No. 1, pp. 117-137, 2012.

[12] H. Taherdoost and A. Keshavarzsaleh, "A Theoretical Review on IT Project Success/Failure Factors and Evaluating the Associated Risks", Mathematical and Computational Methods in Electrical Engineering, Vol.1, pp. 80-88, 2012.

[13] S. Lee and H. Yongm "Agile Software Development Framework in a Small Project Environment", Journal of Indian Prosthodontics Society, Vol. 9, No. 1, pp. 69-88, 2013.
[14] M. Tanner and U. Willingh, "Factors Leading to the Success and Failure of Agile Projects Implemented in Traditionally Waterfall Environments", Journal of Management Research, pp. 693-701, 2014.

[15] K. Silva and S. Santos, "Critical Factors in Agile Software Projects According to People, Process, and Technology Perspective", In: Proc. of Brazilian Workshop on Agile Methods, pp. 4854, 2015.

[16] R. Amponsah and J. Darmoe, "A Study of the Critical Success Factors Influencing Projects in the Ghana Public Sector", International Journal of Business \& Management, pp. 120-132, 2014.

[17] S. Maierhofer, E. Stelzmann, M. Kohlbacher, and B. Fellner, "Requirement Changes and Project Success: The Moderating Effects of Agile Approaches in System Engineering Projects", Verlag Berlin Heidelberg, pp. 60-70, 2010.

[18] T. Chow and D. Cao, "A survey study of critical success factors in agile software projects", Journal of System and Software, Vol.81, pp. 961-971, 2008.

[19] N. Cerpa, M. Bardeen, B. Kitchenham, and J. Verner, "Evaluating logistic regression models to estimate software project outcomes", Information and Software Technology, Vol. 52, pp. 934-944, 2010.

[20] D. Stankovic, V. Nikolicb, M. Djordjevicc, and D. Caod, "A survey study of critical success factors in agile software projects in former Yugoslavia IT companies", Journal of System and Software, Vol. 86, pp. 1663-1678, 2013.

[21] V. Montequin, S. Coquilles, F. Ortega, and J. Villanueva, "Analysis of Success Factors and Failure Causes in Information and Communication Technology (ICT) Projects in Spain", Procedia Technology, Vol. 16, pp. 992 999, 2014.

[22] S. Nguyen, "Success Factors That Influence Agile Software Development Project Success", American Scientific Research Journal for Engineering, Technology and Sciences, Vol. 17, No. 1, pp. 172-222, 2016.

[23] S. Lam, R. Cheung, S. Wong, and E. Chan, "Survey Study of Critical Success Factors in Information System Project Management", International Conference on Internet Studies, Vol. 7, pp. 1-12, 2013.

[24] K. Jammalamadaka and V. Krishna, "Agile Software Development and Challenges", International Journal of Research in Engineering and Technology, Vol. 2, No. 8, pp. 125-129, 2013. 
[25] K. Rao, G. Naidu, and P. Chakka, "A Study of the Agile Software Development Methods, Applicability and Implications in Industry", International Journal of Software Engineering Applications, Vol. 5, No. 2, pp. 35-46, 2011.

[26] P. Serrador and J. K. Pinto, "Does agile work? - A quantitative analysis of agile project success", International Journal of Project Management, Vol. 33, pp. 1040-1051, 2015.

[27] W. Jawaid, T. Jilani, Y. Methmood, and S. Muhammad, "Feature Selection for Agile Development through Data Mining Techniques: An Application", Journal of Applied Environmental and Biological Sciences, Vol.4, pp. 479-489, 2014.

[28] R. Ibrahim, E. Ayazz, S. Nasrmalek, and S. Nakhat, "An Investigation of Critical Failure Factors In Information Technology Projects", Journal of Business and Management, Vol. 10, No. 3, pp. 87-92, 2013.

[29] V. Vithana, S. Fernando, and M. Kapurubandara, "Success Factors for Agile Software Development - A Case Study from Sri Lanka”, International Journal of Computer Applications, Vol. 113, No. 17, pp. 10-18, 2015.

[30] S. Abdalhamid and A. Mishra, "Factors in Agile Methods Adoption", Technology Education Management Journal, Vol. 6, No. 2, pp. 416-421, 2017. 